\title{
Locally Dispersing Populations in Heterogeneous Dynamic Landscapes with Spatiotemporal Correlations. I. Block Disturbance
}

\author{
David E. Hiebeler ${ }^{\mathrm{a}, *}$, Jennifer Houle $^{\mathrm{a}}$, Frank Drummond ${ }^{\mathrm{b}}$, Peter Bilodeau $^{\mathrm{a}}$, \\ Jeffery Merckens ${ }^{\mathrm{a}}$ \\ ${ }^{a}$ Department of Mathematics and Statistics, University of Maine, Orono, ME 04469 \\ ${ }^{b}$ School of Biology and Ecology, 305 Deering Hall, University of Maine, Orono, ME 04469
}

\begin{abstract}
Locally dispersing populations are generally favorably affected by increasing the scale of habitat heterogeneity because they can exploit contiguous patches of suitable habitat. Increasing the spatial scale of landscape disturbances (such as by applying a pesticide to control an unwanted species) drives down population density because of reasons including dispersal-limited recolonization and the resulting increase in temporal variability. Here, we examine how population density changes as the spatial scale of landscape disturbance increases: does it increase due to increases in spatial correlations in landscape habitat type, or does it decrease due to the various spatial and temporal effects of larger-scale disturbances? We use simulations, mean field approximations, pair approximations, landscape-improved pair approximations (LIPA), and block probabilities to investigate a model of a locally dispersing species on a dynamic landscape with spatiotemporally structured heterogeneous habitat. Pesticide is applied at a given spatial scale, leaving habitat unsuitable for some time before dissipating and allowing the habitat to revert to a suitable state. We found that increasing the spatial scale of disturbances (while keeping the overall disturbance rate fixed) can increase population density, but generally only when landscape
\end{abstract}

\footnotetext{
* Corresponding author

Email address: hiebeler@math.umaine.edu (David E. Hiebeler)

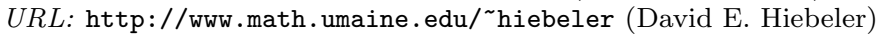

Preprint submitted to Elsevier

July 7, 2016

(C) 2016. This manuscript version is made available under the Elsevier user license http://www.elsevier.com/open-access/userlicense/1.0/ 
turnover is slow relative to population dynamics and when the population is somewhat close to its extinction threshold. Applying control measures at larger spatial scales may allow them to be more effective with the same overall treatment rate. The optimal spatial strategy for applying disturbances depends on both habitat availability as well as the turnover rate of the control measure being used. For the large-scale habitat dynamics in our model, it is possible to analytically calculate spatial correlations in habitat types over arbitrary scales. However, including exact habitat correlations at the triplet scale but approximating population correlations at that scale still neglects information needed to accurately predict simulation results, showing that larger-scale correlations in the population distribution have an important effect on dynamics.

Keywords: habitat disturbance, temporal variability, spatiotemporal structure, stochastic spatial simulation, dynamic habitat

\section{Introduction}

All methods of pest control have a spatial component to them, whether it be the distribution of the release of sterile insects within a given region (Rodríguez, 2015), or allocating removal efforts to patches according to qualities such as patch size or connectivity (Minor and Gardner, 2011). Other non-density dependent sources of mortality, such as lethal temperatures (Torgersen et al., 1999) or pesticide contamination in aquatic systems (Van den Brink et al., 2007) also include important spatial components. Control measures typically lead to spatially heterogeneous distributions of habitat quality which affect fecundity and/or mortality of the target species. The converse is also the case, where conservation of threatened or endangered species likewise has a spatial dynamic (Esler, 2000; Hanski, 2001; Silbernagel, 2003). Habitat loss (Coristine and Kerr, 2011), habitat disturbance (Elkin and Possingham, 2008), range restriction (Channell and Lomolino, 2000), refugia and dispersal corridors for connectivity (Visconti and Elkin, 2009; Cushman and Huettmann, 2010), reintroduction of a species to a locale (Carroll et al., 2003), and climate change 
effects on geographic distribution (Kerr et al., 2015), all have foundations in theoretical spatial ecology. Spatiotemporal variability in habitat quality may arise from several causes, including human activities (Munroe et al., 2002; Jacquemyn et al., 2003; Cerda and Wright, 2004; Masaki, 2004; Linderman et al., 2006; Carrière et al., 2012) or natural events such as the weather (Ringsby et al., 2002) and forest gap formation (Kubo et al., 1996; Manrubia and Solé, 1997).

The expected impacts of heterogeneous habitat vary (Robinson et al., 1992; Oborny, 1994). Movement and dispersal (colonization rate) and extinction rate interact with and are often dependent upon patch quality, patch turnover rate, and habitat fragmentation in affecting the success of a species (Naeem and Colwell, 1991; Amarasekare and Possingham, 2001; Langellotto and Denno, 2001; Fahrig, 2003; Radinger and Wolter, 2015). The spatial scale of correlations in habitat quality relative to organism dispersal distances have been shown to impact population success (Neuhauser, 1998; Lande et al., 1999; Schooley and Branch, 2007). For instance, Liao et al. (2013) demonstrated that populations restricted to local dispersal benefit from habitat clumping whereas those that tend to be characterized by long distance dispersal are not affected by habitat clumping. The same is true of spatial variability in control measures such as when using pesticides to control unwanted species (González-Andujar and Perry, 1995; Gontijo et al., 2013). Increased habitat heterogeneity may also influence variability in population density, as seen in organisms such as desert isopods (Shachak and Brand, 1991; Ye et al., 2014).

In heterogeneous landscapes, habitat association can allow a population to exploit high-quality regions despite the presence of less-suitable habitat (Bolker, 2003; Franken and Hik, 2004; Elkin and Possingham, 2008; Hiebeler et al., 2013). Both the location/connectivity and the quality of habitat patches influence the success of resident or invading species (Ovaskainen and Hanski, 2003; Visconti and Elkin, 2009; Liao et al., 2013). Regarding the geometry of heterogeneity, intermediate sizes of high-quality patches have been found optimal in some population models (North and Ovaskainen, 2007). However, the dynamics of patch quality and size are complex as Mortelliti et al. (2014) show how the landscape 
structure surrounding fragmented high-quality patches can affect population dynamics. At a larger scale, variations in aspects of climate such as precipitation affect community composition, and may drive the spatial distribution of species diversity (Cleland et al., 2013).

The spatial scale of landscape structure, such as autocorrelations of habitat heterogeneity and disturbances, play an important role for populations and communities (Cohen and Levin, 1991; Topping and Sunderland, 1994; Roland and Taylor, 1997; Smith and Gilpin, 1997; Kie et al., 2002; Ripa and Ives, 2003; Pike et al., 2004; Roy et al., 2004; Seabloom et al., 2005; Turner, 2010; Limberger and Wickham, 2012). In particular, the relative scales of disturbance and dispersal are important (Brown and Kodric-Brown, 1977; McCarthy and Lindenmayer, 2000; Johst et al., 2002; Liao et al., 2015), as are the spatial scales of disturbances versus fixed environmental heterogeneities (Kallimanis et al., 2005; Hiebeler and Morin, 2007). The way in which spatial heterogeneity changes over time may help inform management strategies as well (Munguia et al., 2011).

When studying the interactions of temporal and spatial variability, both habitat connectivity and quality of patches have been shown to have smaller influences on patch occupancy in landscapes with dynamic habitat, rather than static habitat distributions (Hodgson et al., 2009). Temporal and spatial variability have also been shown to be inexorably linked (Shachak and Brand, 1991; Harrison and Fahrig, 1995; Moloney and Levin, 1996; Fleishman et al., 2002; Perry, 2002; Cole et al., 2014). One example of this is the basic fact that the temporal scale of observations may affect the spatial variability observed (Hiebeler and Michaud, 2012). In models with spatially correlated disturbances, Johst and Drechsler (2003) show that increasing the spatial correlations usually has a negative effect on population density, in part due to the increase in temporal variability within a given region. The effects of dynamic habitat in spatial population models has been explored in a variety of ways (Iwasa, 1991; Matlack and Monde, 2004; Matlack, 2005; Loehle, 2007; Matlack and Leu, 2007). Model results tend to agree in that the timescale of habitat dynamics rela- 
tive to population dynamics is an essential parameter in determining whether a population persists or goes extinct, even more so than the spatial scale of landscape structure or dynamics (Fahrig, 1992; Keymer et al., 2000; Verheyen et al., 2004; DeWoody et al., 2005). Spatiotemporal variability can also drive the evolution of key parameters such as dispersal (McPeek and Holt, 1992; Roff, 1994), and have effects which are visible in population distributions many years later (Lindborg and Eriksson, 2004).

Here we use a model to explore the effects of spatial and temporal correlations in habitat quality on a locally dispersing population in a dynamic heterogeneous landscape. The model includes disturbance events which simultaneously affect a specified number of contiguous sites, representing control measures (such as a pesticide) applied at a given spatial scale. The primary question we address with this model is: as the spatial scale of disturbance events increases, does population density increase due to the resulting increase in spatial correlations in landscape habitat type (Hiebeler, 2000), or decrease due to the various spatial and temporal effects of larger-scale disturbances (Hiebeler, 2005)? We also investigate how the answer to this question depends on the relative time scales of landscape and population processes.

\section{The Model}

Our model is a spatially discrete continuous-time patch-occupancy Poisson process model on a rectangular lattice with heterogeneous habitat. The habitat type of each site may be either suitable or unsuitable. Suitable sites may either be empty or occupied by the species of interest; unsuitable sites cannot be occupied. Each site may therefore be in one of three states: empty suitable habitat, (empty) unsuitable habitat, or occupied suitable habitat. In our previous similar lattice population models on spatially structured heterogeneous landscapes, the habitat distribution was fixed (Hiebeler, 2000, 2004; Hiebeler and Morin, 2007). Here, the habitat types of sites change over time, as follows. Disturbance events at a given spatial scale cause suitable sites to become unsuitable, representing 
actions such as the application of pesticide, other control measures, or natural events such as gap formation. During such an event, a randomly-chosen $b \times b$ block of sites is simultaneously disturbed, with all suitable sites within the block becoming unsuitable. Any occupied suitable sites that are disturbed in this way also become empty as they instantaneously transition to the unsuitable state. These block disturbances occur at a given location at rate $r_{s u} / b^{2}$, giving a persite disturbance rate of $r_{s u}$ for suitable sites to become unsuitable, regardless of the disturbance size $b$. Note that the time until a particular suitable site is disturbed follows an exponential distribution with a mean of $1 / r_{s u}$; this can be though of as the expected lifetime of a site (Keymer et al., 2000). These block disturbances are like those studied in previous models (Hiebeler, 2005; Hiebeler and Morin, 2007), except that in the previous models, a disturbed site was in principle immediately able to be recolonized, i.e., disturbances had no direct long-term effects (although it generally took some time for a population using only local dispersal to recolonize the center of a large region which had recently been disturbed). In the model used here, each unsuitable site recovers, becoming suitable again, independently at rate $r_{u s}$ as the habitat recovers (e.g., the pesticide dissipates into the environment independent of patch quality, a reasonable assumption due to the dominant role in degradation played by ubiquitous microorganisms(Fenner et al., 2013)). Similar assumptions of correlated disturbances with independent recovery were used in a model of metapopulation persistence by Wilcox et al. (2006). The average time for a disturbed site to become suitable and a candidate for recolonization is therefore $1 / r_{u s}$. Together, the parameters $r_{u s}$ and $r_{s u}$ specify the temporal autocorrelation of habitat type within any given site. The size of the disturbances, given by parameter $b$, determines the spatial autocorrelation of habitat types among adjacent sites at any given time, as derived further below. In addition to the mortality of the species of interest in occupied sites due to the transition of occupied suitable habitat to unsuitable habitat, occupied sites also become empty (suitable) sites due to intrinsic mortality of the species of interest, which occurs at rate $\mu$ per site. Each occupied site in the lattice produces offspring at rate $\phi$. When an 
occupied site produces offspring, one of the four neighboring sites is chosen at random as the target location for its offspring. If the target site is empty and suitable, it is immediately colonized, becoming occupied. This model has similar basic features as that explored by Keymer et al. (2000) and Kun et al. (2009), with the key difference that their models only allowed suitable sites to become unsuitable independently, i.e., their models assumed $b=1$.

When implemented via stochastic simulations, $300 \times 300$ lattices were used, with wraparound boundary conditions. The simulation was first run with only landscape dynamics and no population present, until the landscape was determined to be at equilibrium. Then, a proportion $\rho_{0}$ of the suitable sites were chosen at random to be initially occupied, and the full simulation continued. We used $\rho_{0}=1-\mu / \phi$, the equilibrium for a population with global dispersal at rate $\phi$ and mortality at rate $\mu$ with no unsuitable habitat, as an overestimate of the expected equilibrium population density in this model. If the above expression yielded a value of $\rho_{0}$ less than 0.1 , we began with $10 \%$ of the sites occupied, to avoid fluctuation to extinction due to small initial population size. Simulations were run until equilibrium was reached, with the determination of equilibrium following the ideas of Caswell and Etter (1993), as follows. Every $90,000=300^{2}$ events is considered as one time step. All simulations are run for at least 300 time steps (assuming the population does not go extinct). After that, the population density is recorded after every time step. A linear regression is fitted to the last 100 recorded values (via least squares regression); if the slope of the fitted line has magnitude less than 0.001, the system is considered to be at equilibrium.

Figure 1 shows snapshots of four landscapes with two different proportions of suitable habitat and two different block sizes, demonstrating the types of landscapes included in this model. Figure 2 shows the population on landscapes with the same parameters as the top images in Figure 1. 

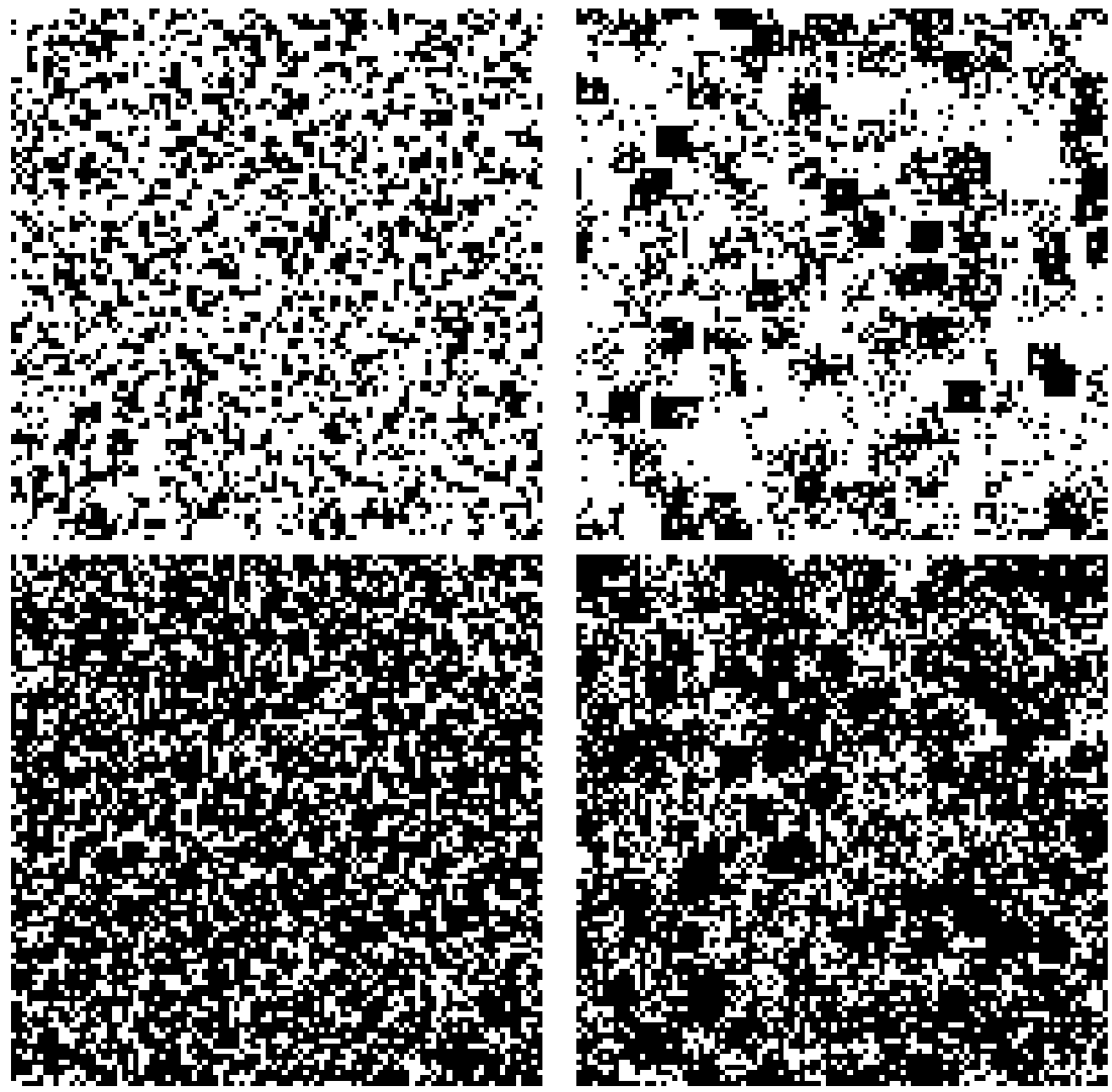

Figure 1: Snapshots of landscapes with four different parameter combinations. White sites are suitable, while black sites are unsuitable. The two landscapes on the top have $r_{s u}=0.5$ and $r_{u s}=1$, giving proportion $p^{*}[s]=2 / 3$ of sites suitable. The two landscapes on the bottom have $r_{s u}=1$ and $r_{u s}=0.5$, giving proportion $p^{*}[s]=1 / 3$ of sites suitable. The two landscapes on the left have disturbance block size $b=2$, while the two landscapes on the right have disturbance size $b=6$, leading to more spatial clustering of habitat types. Landscapes shown here are $100 \times 100$ for visual clarity, though $300 \times 300$ landscapes were used for actual simulations. 

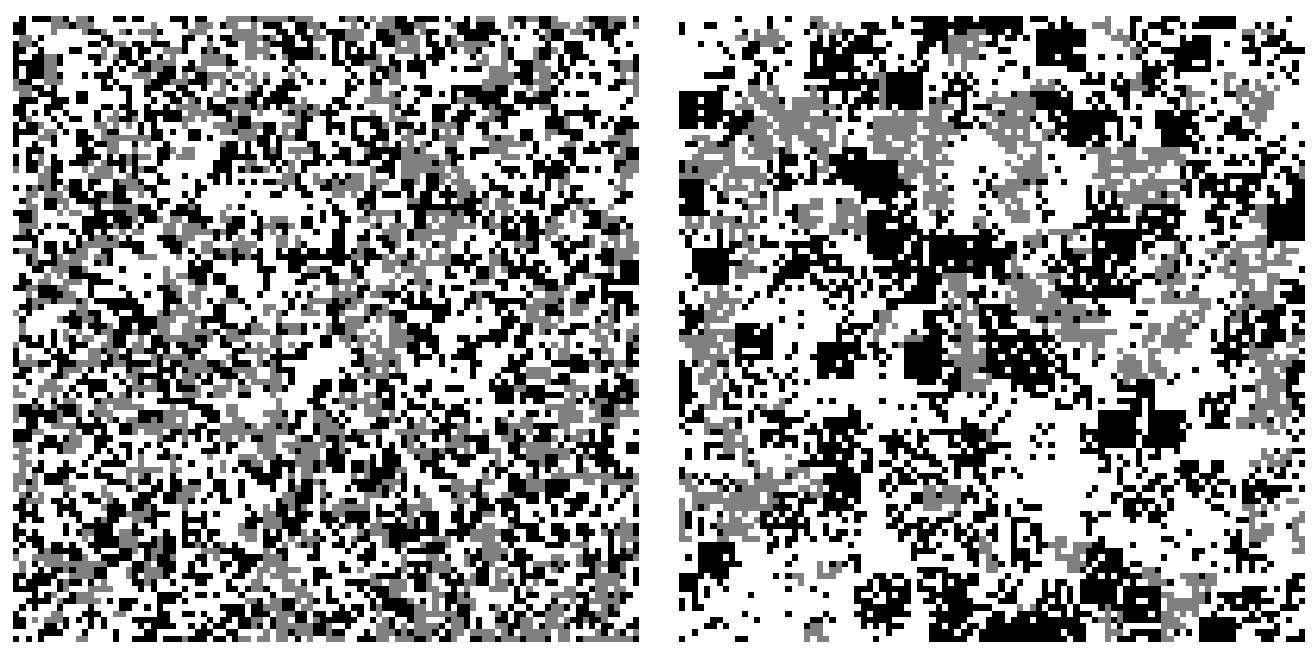

Figure 2: The population on landscapes with $\phi=5, \mu=1, r_{s u}=0.5$, and $r_{u s}=1$ (giving $p^{*}[s]=2 / 3$ ), with disturbance sizes of $b=2$ (left) and $b=6$ (right). White sites are suitable and empty, gray sites are occupied, and black sites are unsuitable.

\subsection{Mathematical approximations}

To represent the state of the system, let the singlet probability $P[i]$ be the probability that a randomly-chosen site is in state $i$, with the states labeled as: $0=$ empty suitable, $1=$ unsuitable, and $2=$ occupied suitable. The doublet probability $P[i j]$ then represents the probability that a pair of randomly-chosen adjacent sites are in states $i$ and $j$, respectively. Triplet probabilities $P[i j k]$ and $2 \times 2$ block probabilities $P\left[\begin{array}{cc}i & j \\ k & l\end{array}\right]$ will similarly be used. The probabilities of all state configurations must sum to one at all block scales:

$$
\sum_{j} P[j]=1, \quad \sum_{i, j} P[i j]=1, \quad \sum_{i, j, k} P[i j k]=1, \quad \sum_{i, j, k, l} P\left[\begin{array}{cc}
i & j \\
k & l
\end{array}\right]=1 .
$$

Marginal (singlet) probabilities can be recovered from double probabilities by summing; similarly, doublet probabilities can be obtained from triplets:

$$
P[i]=\sum_{j} P[i j], \quad P[i j]=\sum_{k} P[i j k]=\sum_{k} P[k i j] .
$$


Partial block probabilities may also be obtained from full blocks:

$$
P\left[\begin{array}{ll}
\cdot & j \\
k & l
\end{array}\right]=\sum_{i} P\left[\begin{array}{ll}
i & j \\
k & l
\end{array}\right],
$$

where the dot in the top-left position indicates that we are not specifying the state of that site. We assume rotational and reflectional symmetry, $P[i j]=$ $P[j i], P[i j k]=P[k j i], P\left[\begin{array}{cc}i & j \\ k & l\end{array}\right]=P\left[\begin{array}{cc}k & i \\ l & j\end{array}\right]=P\left[\begin{array}{ll}j & i \\ l & k\end{array}\right]$, etc.

We will need conditional probabilities of the neighbors of sites being in various states. The quantity $Q_{i \mid j}=P[i j] / P[j]$ represents the conditional probability that, if we first randomly choose a site in state $j$, then a randomly-chosen neighbor of that site is in state $i$. Similarly, $Q_{i \mid j k}=P[i j k] / P[j k]$ represents the probability that, if we randomly choose a triplet of adjacent sites with the second and third sites in states $j$ and $k$, respectively, then the first site is in state $i$.

We sometimes describe probabilities involving only the landscape configuration. To distinguish them from probabilities describing the full system, we use lowercase $p$ to refer to these probabilities. The proportions of suitable (state $=$ 0 or 2 ) and unsuitable $($ state $=1)$ sites are $p[s]=1-P[1]$ and $p[u]=P[1]$, respectively, and similarly we use doublet probabilities $p[s s], p[s u]$, and so on. We use lowercase $q$ for conditional probabilities involving neighbors, such as $q_{s \mid s}=p[s s] / p[s]$ giving the probability that a particular neighbor of a randomlychosen suitable site is also suitable.

We can also relate landscape-only probabilities to probabilities from the full model including the population, by considering whether suitable sites are empty or occupied. In particular, two relations that will be used when deriving our ODEs are

$$
\begin{aligned}
p[s u] & =p[s]\left(1-q_{s \mid s}\right)=P[01]+P[21] \\
p[s s] & =p[s] q_{s \mid s}=P[00]+P[02]+P[20]+P[22] \\
& =P[00]+2 P[02]+P[22] .
\end{aligned}
$$


The proportion of suitable sites that are occupied will be written as $\rho=$ $P[2] / p[s]$. Another quantity that will arise in the subsequent analysis is $\theta$, defined as the probability that when one site in a doublet is disturbed, the other site is simultaneously affected by the disturbance. Following Hiebeler (2005), this quantity may be related to the disturbance block size $b$ via the expression $\theta=1-1 / b$, derived by summing over all locations a disturbance event could be aimed at which would affect both sites in the pair. The rate at which disturbance events affect one or both sites in a doublet can similarly be derived as $r_{s u}(2-\theta)$.

\subsection{Landscape dynamics}

To simplify analysis, we assume that population dynamics take place after the landscape has reached a dynamic equilibrium, i.e., the amount $p[s]$ and spatial clustering $q_{s \mid s}$ of suitable and unsuitable sites have reached steady-state values, even as sites continue to transition between suitable and unsuitable habitat states.

Note that for the dynamics of habitat types in the landscape, none of the transitions between suitable and unsuitable habitat depend on the habitat types of neighboring sites. That is, a site may change its habitat type at any time, regardless of the states of its neighbors. Because of this, spatial correlations of habitat types do not grow over arbitrarily large distances in the lattice, as they do in the population occupancy status of sites in models with localized dispersal (Levin and Durrett, 1996). As a result, no moment-closure approximations are needed when writing ODEs to describe landscape dynamics.

Equations for landscape dynamics are given in Appendix A.1. From Equations (A.2) and (A.4), the proportions of sites with suitable and unsuitable habitat, as well as the clustering of suitable sites at equilibrium, can be expressed 


$$
\begin{aligned}
p^{*}[s] & =\frac{r_{u s}}{r_{u s}+r_{s u}} \\
p^{*}[u] & =\frac{r_{s u}}{r_{u s}+r_{s u}} \\
q_{s \mid s}^{*} & =\frac{p^{*}[s s]}{p^{*}[s]}=\frac{r_{u s}}{r_{u s}+r_{s u}(1-\theta / 2)} .
\end{aligned}
$$

Because $0 \leq \theta \leq 1$, the clustering satisfies $q_{s \mid s}^{*} \geq p^{*}[s]$, with equality if and only if $r_{u s}=0$ (a degenerate case where unsuitable sites never recover to suitable status) or $\theta=0$ (corresponding to $b=1$, where suitable sites are disturbed and become unsuitable individually, i.e. multiple sites are never simultaneously disturbed, and so no spatial correlations in habitat types develop over time). As $\theta \rightarrow 1$, i.e. disturbances occur at larger and larger spatial scales (but less and less frequently to control the per-site disturbance rate), then $q_{s \mid s}^{*} \rightarrow r_{u s} /\left(r_{u s}+\right.$ $\left.r_{s u} / 2\right)=2 /\left(1+1 / p^{*}[s]\right)$. This establishes the following bounds on clustering of suitable sites at equilibrium:

$$
p^{*}[s] \leq q_{s \mid s}^{*} \leq \frac{2}{1+1 / p^{*}[s]} .
$$

Finally, note that given a particular disturbance rate $r_{s u}$, Equation (6) may be solved for

$$
r_{u s}=\frac{r_{s u} p^{*}[s]}{1-p^{*}[s]},
$$

giving the recovery rate needed to achieve a specified level of habitat availability.

\subsection{Singlet equations}

A system of ordinary differential equations for $P[0], P[1]$, and $P[2]$ may be written to describe the proportions of sites in various states in the full population model. By the assumption of landscape equilibrium, $P[1]=p^{*}[u]$ is fixed at the value given by Equation (7), and because $P[0]=1-P[2]-p^{*}[u]$ by Equation (1), in fact we only need a single ODE to characterize the system:

$$
\frac{d P[2]}{d t}=P[0] \phi Q_{2 \mid 0}-P[2]\left(\mu+r_{s u}\right) .
$$


We then use the mean-field approximation that the states of all sites are independent/uncorrelated, i.e. $Q_{i \mid j}=P[i]$, to approximate $Q_{2 \mid 0}$ by $P[2]$ in the above ODE. This closed system then has equilibrium

$$
P^{*}[2]=p^{*}[s]-\frac{\mu+r_{s u}}{\phi},
$$

with $p^{*}[s]$ given by Equation (6). The equilibrium proportion of suitable sites that are occupied is $\rho^{*}=P^{*}[2] / p^{*}[s]=1-\left(\mu+r_{s u}\right) /\left(\phi p^{*}[s]\right)$. This result agrees with the standard form of mean-field results for population models (e.g., Keymer et al., 2000; Hiebeler and Morin, 2007), i.e., the proportion of all sites occupied is the proportion of suitable sites less the total disturbance rate divided by the reproduction rate (with the reproduction rate modified to filter out offspring that land on unsuitable sites).

\subsection{Doublet equations}

Next, we include additional information about spatial correlations by studying ODEs for doublet probabilities $P[i j]$.

The following self-contained set of ODEs for the population dynamics on the landscape follow from Equations (A.10)-(A.12), derived in Appendix A.2 using ordinary pair approximation:

$$
\begin{aligned}
\frac{d P[00]}{d t}= & 2 p^{*}[s] r_{u s}-2 P[2] r_{u s}+2 P[02]\left(\mu-r_{u s}\right) \\
& -P[00]\left(\frac{2(z-1) P[02] \phi}{z P[0]}+r_{s u}(2-\theta)+2 r_{u s}\right) \\
\frac{d P[01]}{d t}= & P[00] r_{s u}(1-\theta)+\left(1+p^{*}[s]\left(q_{s \mid s}^{*}-2\right)\right) r_{u s}+P[02](1-\theta) r_{s u} \\
& +\left(p^{*}[s]\left(1-q_{s \mid s}^{*}\right)-P[01]\right) \mu \\
& -P[01]\left(r_{u s}+r_{s u}+\frac{z-1}{z} \phi \frac{P[02]}{P[0]}\right) \\
\frac{d P[02]}{d t}= & P[00]\left(\frac{(z-1) P[02] \phi}{z P[0]}+r_{u s}-\mu\right)+P[2] r_{u s}+p^{*}[s] q_{s \mid s}^{*}\left(\mu-r_{u s}\right) \\
& -P[02]\left(\frac{(z-1) P[02] \phi}{z P[0]}+\frac{\phi}{z}+r_{s u}(2-\theta)-r_{u s}+3 \mu\right)
\end{aligned}
$$

${ }_{261}$ with $p^{*}[s]$ and $q_{s \mid s}^{*}$ given by Equations (6) and (8). 


\subsection{Landscape-improved doublet equations}

Additional information about the spatial structure could be included in the model by using triplet equations for $P[i j k]$ with $i, j, k \in\{0,1,2\}$. However, the number of state variables would be $27=3^{3}$, though some reduction would be possible by the assumptions of symmetry and of the landscape being at equilibrium. Instead, we use another approach we refer to as landscape-improved pair approximation (LIPA). The LIPA incorporates known information about the structure of habitat at larger (triplet) scales while still using pair approximation for the population structure, as a straightforward generalization of improved mean-field approximations previously used (Hiebeler, 2007; Hiebeler and Morin, 2007).

The ODEs, derived in Section Appendix A.3, are:

$$
\begin{aligned}
\frac{d P[00]}{d t}= & 2 P[01] r_{u s}+2 P[02] \mu \\
& -P[00]\left(r_{s u}(2-\theta)+2 \frac{z-1}{z} \phi \frac{\left(p^{*}[s s]-p^{*}[u s s]\right) P[02]}{p^{*}[s s](P[00]+P[02])}\right) \\
\frac{d P[01]}{d t}= & (P[00]+P[02]) r_{s u}(1-\theta)+P[11] r_{u s}+P[21] \mu \\
- & P[01]\left(r_{u s}+r_{s u}+\frac{z-1}{z} \phi \frac{p^{*}[u s s] P[02]}{\left(p^{*}[s]-p^{*}[s s]\right)(P[00]+P[02])}\right) \\
\frac{d P[02]}{d t}= & \frac{z-1}{z} P[00] \phi \frac{\left(p^{*}[s s]-p^{*}[u s s]\right) P[02]}{p^{*}[s s](P[00]+P[02])}+P[12] r_{u s}+P[22] \mu \\
- & P[02]\left(\mu+r_{s u}(2-\theta)+\frac{\phi}{z}+\frac{z-1}{z} \frac{\left(p^{*}[s s]-p^{*}[u s s]\right) P[02]}{p^{*}[s s](P[00]+P[02])}\right)
\end{aligned}
$$

with $p^{*}[s], p^{*}[s s], p^{*}[u s s]$ given by Equations (6), (A.4), and (A.9), respectively.

\subsection{Block equations}

Finally, we also explored the behavior of the model by considering the rates of change of all possible configurations of $2 \times 2$ blocks of sites. There are $3^{4}=81$ probabilities in total, although assumptions of symmetry and of the landscape being at equilibrium again reduce this. There are too many equations to include here explicitly; a derivation of the process used to derive the equations is given in Section Appendix A.4. A moment-closure approximation similar to that used 
in pair approximation was again used to study this system, where the states of non-adjacent sites were assumed to be conditionally independent, if the state of an intermediate common neighbor was known.

\section{Results}

We first performed two parameter sweeps as the size of disturbances increased from $1 \times 1$ disturbances (i.e., sites independently disturbed) up to $10 \times 10$ disturbances. Figure 3 shows how $\rho^{*}$, the proportion of suitable sites occupied at equilibrium, varies as the disturbance size $b$ increases. Both parameter sweeps were done with $r_{s u}=r_{u s}$, resulting in $50 \%$ of the sites in the landscape being suitable, and with $\phi=10$ and $\mu=1-r_{s u}$, giving a total disturbance rate (from both intrinsic mortality and habitat disturbance) of $\mu+r_{s u}=1$ per occupied site. The left half of the figure had very slow landscape dynamics $\left(r_{s u}=0.02\right)$, while the right half had faster landscape dynamics $\left(r_{s u}=1\right)$. When landscape turnover is very slow relative to population dynamics (dispersal and population growth), increasing the spatial scale of disturbance blocks can increase population density, due to the large contiguous regions of suitable habitat that exist with larger disturbances, which the locally dispersing population can then more easily exploit. Eventually, however, with large enough disturbances, the spatial and temporal correlations that arise drive the equilibrium population density back down again. When landscape turnover is more rapid, no initial increase in population density is seen as the size of disturbance events increases.

To determine whether the behavior seen in Figure 3 depended on the amount of suitable habitat, we performed a triple parameter sweep. The values of landscape parameters $r_{s u}$ and $r_{u s}$ were independently varied from 0.02 to 1 with 50 uniformly-spaced values. For each combination of values, the disturbance size $b$ was varied from 1 to 10 . For simulation results, 10 independent replicate simulations were performed for each parameter combination (giving a total of $2.5 \times 10^{5}$ simulations), and the values of $\rho^{*}=P^{*}[2] / p^{*}[s]$ were averaged among the 10 replicates. The value of $b$ that maximized $\rho^{*}$ for each pair of values $r_{s u}$, 

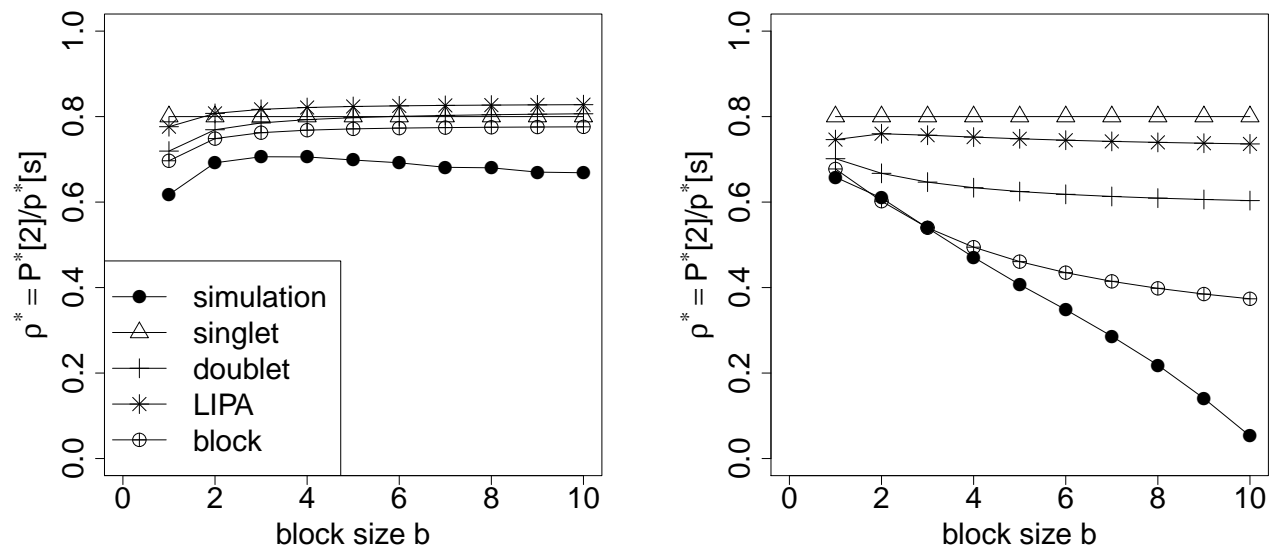

Figure 3: The proportion of suitable sites occupied, $\rho=P[2] / p[s]$, is shown as the size $b$ of disturbance blocks $b$ varies from 1 to 10 . Both landscapes have $50 \%$ suitable habitat, with $r_{s u}=r_{u s}$. Left: $r_{s u}=0.02$ (slower landscape dynamics). Right: $r_{s u}=1$ (faster landscape dynamics). Simulation results are the average of 10 replicate simulations for each value of $b$; error bars are not shown because the largest sample standard deviation for the value of $\rho$ observed among the replicate simulations for any particular value of $b$ was only roughly 0.02 . 
$r_{u s}$ was recorded (the value 0 was used if the population went extinct for all values of $b$ ). The results are shown in the heat maps in Figure 4. Along a line of constant slope $m$ from the origin, $r_{s u}=m r_{u s}$, giving a constant proportion $p^{*}[s]=1 /(1+m)$ of suitable habitat. As one travels away from the origin on such a line, the turnover rate of habitat increases, driving population density down until it eventually goes extinct. Simulation results in Figure 4 show that in general, population density is maximized with larger block sizes when the amount of suitable habitat available has decreased nearly to the critical value at which the population goes extinct. None of the mathematical approximations accurately predict the simulation results, though as more detailed spatial information is included in the approximations (from pair approximation to LIPA to block probabilities), the results begin to come closer to matching simulation results. This suggests that the correlation structure in the landscape at larger spatial scales that arises from the interactions of local dispersal and landscape structure is still playing an important role in the dynamics, and that including exact correlations in habitat type at the triplet scale together with pair approximation of population correlations at that scale is still not sufficient to accurately predict the behavior of the model. Note that for cases where the population density was maximized for a block size of 10, additional exploration of the model suggests that population density does not begin to decrease even up to $b=20$. Results are presented with a maximum value of $b=10$ here for more clarity in the color bar figure legends.

Figure 5 shows a similar parameter sweep, but with $r_{s u}$ and $r_{u s}$ varying between 0.02 and 4 (again with 50 uniformly-spaced values). At this sampling resolution of the parameters, the image showing simulation results did not encounter values of $r_{u s}$ and $r_{s u}$ for which population density was maximized with $b=10$, even though such cases were seen in Figure 4 . However, the band of parameter values for which this happens is too narrow to be seen in Figure 5. Note that the color scale for the simulation results in Figure 5 was adjusted for clarity.

In our model, decreasing the amount of suitable habitat without changing the 


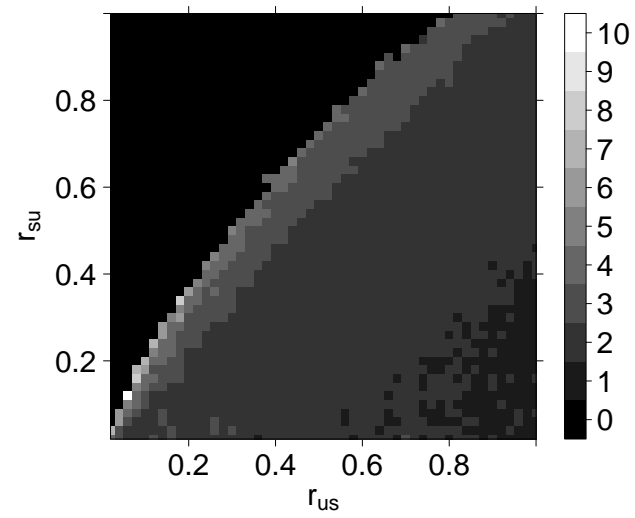

Simulations

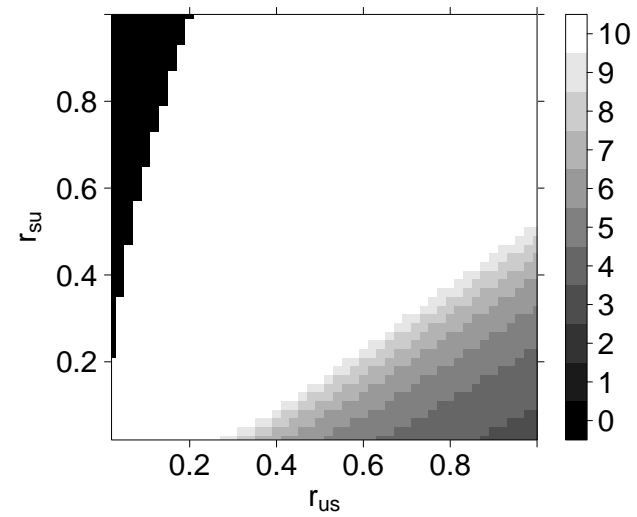

LIPA Doublet

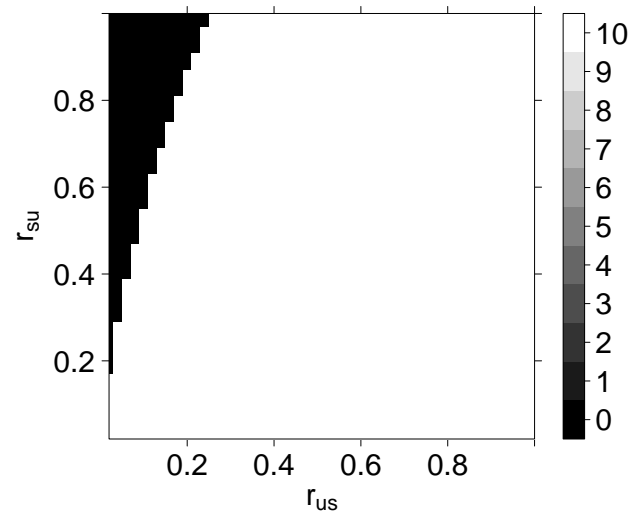

Doublet

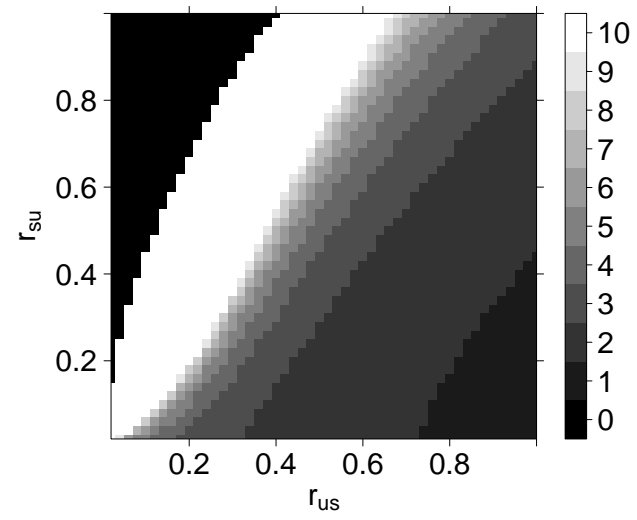

Block

Figure 4: Heat maps showing the block size $b$ that maximizes population density $\left(\rho^{*}\right)$, as a function of landscape parameters $r_{u s}$ and $r_{s u}$ which vary between 0 and 1 . Results are shown for simulations, as well as the various approximations used here. 


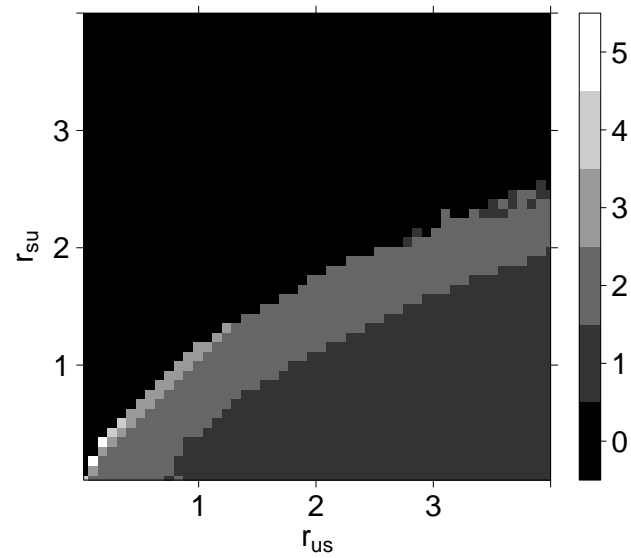

Simulations

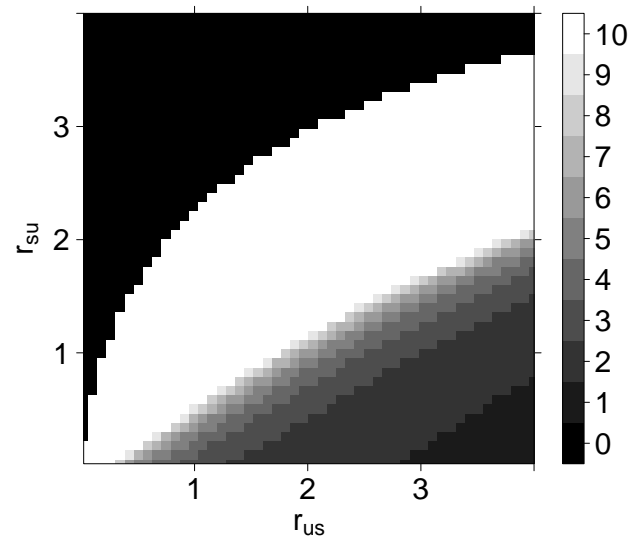

LIPA Doublet

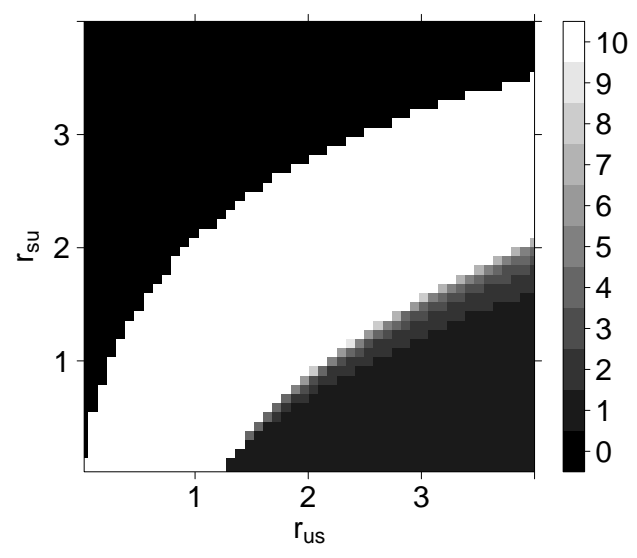

Doublet

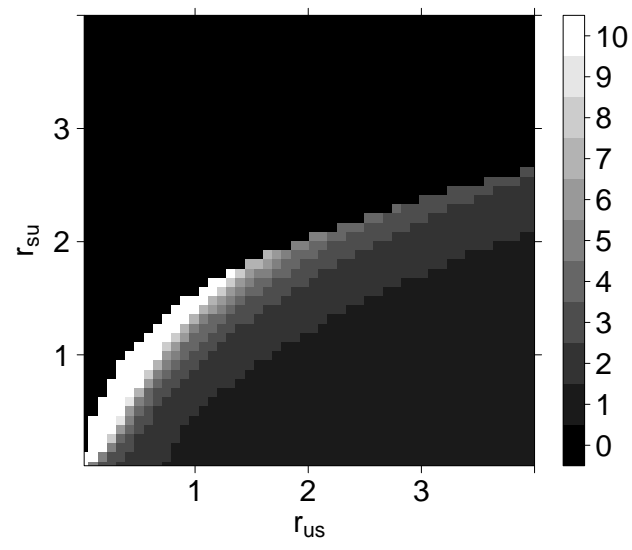

Block

Figure 5: Heat maps showing the block size $b$ that maximizes population density $\left(\rho^{*}\right)$, with landscape parameters $r_{u s}$ and $r_{s u}$ now varying between 0 and 4 . Note that simulation results have a different color scale to make results more clearly visible, as the largest block size $b$ which maximized population density for all parameters shown here was $b=5$. 
average lifetime of a patch $\left(1 / r_{s u}\right)$ or the total mortality of occupied sites (the sum of intrinsic mortality $\mu$ and disturbance rate $r_{s u}$ ) corresponds to moving horizontally to the left across either Figure 4 or 5 . Increasing the turnover rate of habitat, while maintaining the same amount of suitable habitat, corresponds to moving along a line of fixed slope away from the origin. As can be seen in those two figures, which change will have the largest effect, such as leading the system to extinction most easily, depends on where one begins in the parameter space. Species close to the extinction threshold (near the top-left border of the non-black region in the figures) can likely tolerate a greater increase in habitat turnover (moving out along a line from the origin) than a corresponding reduction of suitable habitat (moving to the left) before moving into the black extinction region of the parameter space. For species far from the extinction threshold (farther lower right in the figures), the opposite can be true.

\section{Discussion}

In agreement with Keymer et al. (2000), we found that a population may go extinct if either there is insufficient suitable habitat available (far up in Figure 5, with large $r_{s u}$ relative to $r_{u s}$ ), or if the habitat turnover rate is too rapid relative to population demographic rates (far out from the origin along a line with fixed slope in Figure 5). The specific cause of extinction will depend upon the population dynamics / life history strategy of the species. Our findings suggest that an $r$-strategist species is more likely to be adversely affected by insufficient suitable habitat, whereas a $K$-strategist is more likely to be negatively affected by a high turnover rate (under the assumption of only local dispersal). Johst et al. (2002) found the same results, but also showed that in a dynamic landscape, the most important factor that affects long-term population persistence is the ability of individuals to disperse long-distance.

In addition, we have investigated the spatial scale of disturbances, and the resulting effects on correlations in habitat type and clustering of occupied sites, as another factor affecting survival of the species of interest. The effects of the 
disturbance size $b$ vary with both the amount of suitable habitat and turnover rate (or patch lifetime, in the terms of Keymer et al. (2000)).

The ultimate goal of taking advantage of spatial structure in control measures (such as pesticides) of invasive species is to find a way to make those control measures more effective. That is, can certain ways of spatially and temporally allocating treatments allow one to reduce the density of an invasive species more greatly, for a given amount of effort?

The results seen in this model indicate that when the population is far from its critical point, i.e., when the invasive species is thriving, increasing the spatial scale of control measures may be more effective. This involves applying the pesticide at the same total rate, but in less frequent but larger-scale patterns. In that case, the direct effects of larger disturbances on the population outweigh the more highly clustered distribution of suitable habitat that arises, since population density is maximized with smaller disturbance sizes. Liao et al. (2013), while not investigating the size of a block disturbance, did find that random habitat degradation or disturbance posed a more serious threat to species persistence than clustered habitat degradation. However, as a pest population is pushed more closely to its critical extinction point, smaller spatial scale disturbances are more effective at reducing population density. In this case, the benefits of the increased clustering of suitable habitat outweigh the direct effects of large-scale disturbances on the population, since population density is maximized with larger disturbance sizes. Increasing the rate of pesticide temporal frequency would be a better strategy in that case, in order to further reduce population density. Our findings suggest that "area-wide" management of invasive species (strategy of large regional block applications of insecticides) may need to be rethought (Vreysen et al., 2007) and become more flexible depending upon a dynamic understanding of the species critical extinction point.

Keymer et al. (2000) found that in ephemeral landscapes (i.e., those with large values of $r_{s u}$ ), changes in patch lifetime via the disturbance rate $r_{s u}$ had a larger effect than changing the amount of suitable habitat, while in virtually permanent environments (with small $r_{s u}$ ), habitat availability played a larger 
role. Their results were based in part upon mean-field approximations, and examining the rates of change $\partial \rho / \partial \bar{\tau}$ (where $\bar{\tau}=1 / r_{s u}$ ) and $\partial \rho / \partial p^{*}[s]$ in our notation. Mean-field approximations are inadequate for studying our system due to its neglect of all spatial structure, and closed-form analytical solutions are not available for our more detailed approximations. Also, we chose to characterize our system via the landscape parameters $r_{s u}$ and $r_{u s}$, rather than via what we consider a dependent variable $p^{*}[s]$. This difference can change quantitative results such as the magnitude of the change in population density with respect to model parameters mentioned above because of the nonlinear relation between $p^{*}[s]$ and $r_{u s}$ in Equations (6) and (9), which give $d p^{*}[s] / d r_{u s}=\left(1-p^{*}[s]\right) /\left(r_{u s}+\right.$ $\left.r_{s u}\right)$ and $d r_{u s} / d p^{*}[s]=r_{s u} /\left(1-p^{*}[s]\right)^{2}$. However, our qualitative results are generally in agreement with those of Keymer et al. (2000), though with the additional complexity of the spatial scale of disturbances included.

Other studies of the effects of spatially correlated disturbances found that more highly-correlated disturbances tended to reduce population density or the effective speed at which a population spreads in a variety of cases (Johst and Drechsler, 2003; Brown et al., 2012). Liao et al. (2015) also found that increasing the spatial scale or correlation of disturbance lowered the population density of locally dispersing species under a variety of conditions such as varying intraspecific competition intensities and relative birth rates. Indeed, as seen in our Figure 3, population density does decrease with increased disturbance size more often than it increases, though sometimes by relatively minor amounts depending on the relative timescales of landscape dynamics and population dynamics. This is also reflected in Figures 4 and 5 which show that population density is maximized for small block sizes over the majority of the landscape-dynamics parameter space. It is possible that had Johst and Drechsler (2003) explored their parameter space more thoroughly, they would also have seen an increase in population density as disturbances become more spatially correlated, particularly near the extinction threshold. In our model, the lasting effects on suitable habitat distribution resulting from disturbances also allows for larger-scale disturbances to increase population density, particularly when habitat turnover is 
relatively slow compared with the population's demographic rates.

In the model of Kun et al. (2009), which is similar to ours but with individualsite disturbances $(b=1)$, they found that equilibrium population density was maximized at an intermediate temporal disturbance rate ( $r_{s u}$ in our notation), as long as intrinsic mortality ( $\mu$ in our notation) was not 0 . This is because as the disturbance rate $r_{s u}$ increases from a small initial value, there is initially a direct effect on the population where more occupied sites become empty because they are disturbed. However, when the disturbance rate becomes large enough, it causes pathways to open up between patches of suitable habitat, allowing recolonization of small patches that have gone extinct. We see a similar effect with the spatial scale of disturbances in our model. Further exploration of our model indicates that when $\mu=0$, increasing the scale $b$ of disturbances drives equilibrium population density $\rho^{*}$ down, but that when $\mu>0$, increasing $b$ initially increases $\rho^{*}$ (but then possibly subsequently decreasing $\rho^{*}$ for large enough $b$, particularly for larger landscape turnover rates). In a model with large-scale disturbances which do not have lingering effects on habitat type, Banitz et al. (2008) found that intermediate disturbance rates maximize biodiversity. Results presented in their Figure 3 also indicate that for some disturbance rates, biodiversity is maximized for intermediate spatial scales of disturbances (with scale quantified by the number of clumps a given large-scale disturbance event is broken into).

There are of course many other aspects of spatiotemporal variability that may come into play in population and community dynamics. A model with more complex disturbance dynamics showed that temporal autocorrelations among disturbances had a larger influence than features such as spatial autocorrelation or separate disturbances or the spatial scale of individual disturbances (Moloney and Levin, 1996). The interactions between spatial and temporal variability may impact competition between multiple species (Chesson, 1985), while the spatial scale of fragmentation also impacts the amount of time (and therefore the chances of success) for dispersing organisms to find new suitable habitat (Doak et al., 1992). In general, the dispersal behavior of organisms is 
another key factor in determining how the spatiotemporal structure of dynamic landscapes will be perceived by a given organism (Matlack and Monde, 2004). Including heterogeneity in habitat turnover rates across a landscape also affects the connectivity of patches of suitable habitat to an extent that population dynamics may be noticeably affected (Matlack and Leu, 2007).

In summary, our key findings are as follows:

1. When landscape turnover is very slow relative to population dynamics, increasing the spatial scale of disturbance blocks can increase population density.

2. However, with large enough disturbances, the spatial and temporal correlations that arise drive the equilibrium population density down.

3. When landscape turnover is more rapid, no initial increase in population density is seen as the size of disturbance events increases and subsequently population size continues to decrease.

4. In general, population density is maximized with larger block sizes when the amount of suitable habitat available has decreased nearly to the critical value at which the population goes extinct. This suggests that the correlation structure in the landscape at large spatial scales that arises from the interactions of local dispersal and landscape structure is still playing an important role in the dynamics.

5. Species close to their extinction threshold tolerate a greater increase in habitat turnover than a corresponding reduction of suitable habitat before eventual extinction.

6. For species far from their extinction threshold, the opposite appears to be true. In this case, the direct effects of larger disturbances on the population outweigh the more highly clustered distribution of suitable habitat, and population density is maximized with smaller disturbance sizes.

7. However, as a pest population is pushed more closely to its critical extinction point, smaller spatial scale disturbances are more effective at reducing population density. In this case, the benefits of the increased clustering 
of suitable habitat outweigh the direct effects of large-scale disturbances on the population, since population density is maximized with larger disturbance sizes. Increasing the rate of pesticide temporal frequency would be a better strategy in that case, in order to further reduce population density.

Applied perspectives of our findings suggest spatial strategies for managing dynamic habitat may be helpful not only for controlling invasive or unwanted species, but also in protecting desired species in managed landscapes, by considering the spatial and temporal distribution of refugia (Topping and Sunderland, 1994). When controlling invasive species, we typically would like to apply control measures, such as pesticides, infrequently for economic, ecological, or environmental reasons (Terry and Gourley, 2010). However, decisions about the optimal spatial scale for control measures should also be considered in concert with decisions about the temporal scale. As seen here, such decisions may need to be modified on the go, because the optimal strategy may vary as the population being controlled approaches local extinction in the region of interest. Our findings have great implications in pest management. The philosophy of integrated pest management (IPM) has for a long time been based upon making management decisions (usually implementation of pesticide applications) on "real-time" pest densities(Pedigo and Rice, 2014). However, this has almost entirely been within a framework of temporal pesticide frequency of applications and not a more integrated synthesis of both temporal and spatial dynamics of disturbance.

\section{Acknowledgements}

This material is based upon work supported by the National Science Foundation under Grant No. DMS-0718786 to D.H. We dedicate this paper to the memory of Charlie Slavin, and are grateful for all his help. 


\section{References}

Amarasekare, P., Possingham, H., 2001. Patch dynamics and metapopulation theory: The case of successional species. Journal of Theoretical Biology 209, 333-344.

Banitz, T., Huth, A., Grimm, V., Johst, K., 2008. Clumped versus scattered: How does the spatial correlation of disturbance events affect biodiversity? Theoretical Ecology 1, 231-240.

Bolker, B. M., 2003. Combining endogenous and exogenous spatial variability in analytical population models. Theoretical Population Biology 64, 255-270.

Brown, J. H., Kodric-Brown, A., 1977. Turnover rates in insular biogeography: Effect of immigration on extinction. Ecology 58, 445-449.

Brown, R., James, A., Pitchford, J., Plank, M., 2012. Habitat fragmentation: Simple models for local persistence and the spread of invasive species. Journal of Theoretical Biology 310, 231-238.

Carrière, Y., Ellers-Kirk, C., Hartfield, K., Larocque, G., Degain, B., Dutilleul, P., Dennehy, T. J., Marsh, S. E., Crowder, D. W., Li, X., Ellsworth, P. C., Naranjo, S. E., Palumbo, J. C., Fournier, A., Antilla, L., Tabashnik, B. E., 2012. Large-scale, spatially-explicit test of the refuge strategy for delaying insecticide resistance. PNAS 109 (3), 775-780.

Carroll, C., Phillips, M. K., Schumaker, N. H., Smith, D. W., Apr. 2003. Impacts of landscape change on wolf restoration success: Planning a reintroduction program based on static and dynamic spatial models. Conservation Biology $17(2), 536-548$.

Caswell, H., Etter, R. J., 1993. Ecological interactions in patchy environments: From patch occupancy models to cellular automata. In: Levin, S., Powell, T., Steele, J. (Eds.), Patch Dynamics. Springer-Verlag, pp. 93-109. 
Cerda, H., Wright, D., 2004. Modeling the spatial and temporal location of refugia to manage resistance in $B t$ transgenic crops. Agriculture, Ecosystems, and Environment 102, 163-174.

Channell, R., Lomolino, M., 2000. Dynamic biogeography and conservation of endangered species. Nature 403, 84-86.

Chesson, P. L., 1985. Coexistence of competitors in spatially and temporally varying environments: A look at the combined effects of different sorts of variability. Theoretical Population Biology 28, 263-287.

Cleland, E. E., Collins, S. L., Dickson, T. L., Farrer, E. C., Gross, K. L., Gherardi, L. A., Hallett, L. M., Hobbs, R. J., Hsu, J. S., Turnbull, L., Suding, K. N., 2013. Sensitivity of grassland plant community composition to spatial vs. temporal variation in precipitation. Ecology 94 (8), 1687-1696.

Cohen, D., Levin, S. A., 1991. Dispersal in patchy environments: The effects of temporal and spatial structure. Theoretical Population Biology 39, 63-99.

Cole, E. M., Bustamante, M. R., Almeida-Reinoso, D., Funk, W. C., 2014. Spatial and temporal variation in population dynamics of Andean frogs: Effects of forest disturbance and evidence for declines. Global Ecology and Conservation $1,60-70$.

Coristine, L., Kerr, J., 2011. Habitat loss, climate change, and emerging conservation challenges in Canada. Canadian Journal of Zoology 89 (5), 435-452.

Cushman, S., Huettmann, F. (Eds.), 2010. Spatial Complexity, Informatics, and Wildlife Conservation. Springer.

DeWoody, Y. D., Feng, Z., Swihart, R. K., 2005. Merging spatial and temporal structure within a metapopulation model. The American Naturalist 166 (1), $42-55$

Doak, D. F., Marino, P. C., Kareiva, P. M., 1992. Spatial scale mediates the influence of habitat fragmentation on dispersal success: Implications for conservation. Theoretical Population Biology 41, 315-336. 
Elkin, C. M., Possingham, H., 2008. The role of landscape-dependent disturbance and dispersal in metapopulation persistence. The American Naturalist $172(4), 563-575$.

Esler, D., Apr. 2000. Applying metapopulation theory to conservation of migratory birds. Conservation Biology 14 (2), 366-372.

Fahrig, L., 1992. Relative importance of spatial and temporal scales in a patchy environment. Theoretical Population Biology 41, 300-314.

Fahrig, L., 2003. Effects of habitat fragmentation on biodiversity. Annual Review of Ecology and Systematics 34, 487-515.

Fenner, K., Canonica, S., Wackett, L. P., Elsner, M., 2013. Evaluating pesticide degradation in the environment: Blind spots and emerging opportunities. Science 341 (6147), 752-758.

Fleishman, E., Ray, C., Sjögren-Gulve, P., Boggs, C. L., Murphy, D. D., Jun. 2002. Assessing the roles of patch quality, area, and isolation in predicting metapopulation dynamics. Conservation Biology 16 (3), 706-716.

Franken, R. J., Hik, D. S., 2004. Influence of habitat quality, patch size and connectivity on colonizaiton and extinction dynamics of collared pikas Ochotona collaris. Journal of Animal Ecology 73, 889-896.

Gontijo, P., Picanço, M., Pereira, E., Martins, J., Chediak, M., Guedes, R., 2013. Spatial and temporal variation in the control failure likelihood of the tomato leaf miner, Tuta absoluta. Annals of Applied Biology 162 (1), 50-59.

González-Andujar, J., Perry, J., 1995. Models for the herbicidal control of the seed bank of avena sterilis: the effects of spatial and temporal heterogeneity and of dispersal. Journal of Applied Ecology 32, 578-587.

Hanski, I., 2001. Spatially realistic theory of metapopulation ecology. Naturwissenschaften $88,372-381$. 
Harrison, S., Fahrig, L., 1995. Landscape pattern and population conservation. In: Hansson, L., Fahrig, L., Merriam, G. (Eds.), Mosaic Landscapes and Ecological Processes. Chapman \& Hall, Ch. 12, pp. 293-308.

Hiebeler, D. E., 2000. Populations on fragmented landscapes with spatially structured heterogeneities: Landscape generation and local dispersal. Ecology 81 (6), 1629-1641.

Hiebeler, D. E., 2004. Competition between near and far dispersers in spatially structured habitats. Theoretical Population Biology 66 (3), 205-218.

Hiebeler, D. E., 2005. Spatially correlated disturbances in a locally dispersing population model. Journal of Theoretical Biology 232 (1), 143-149.

Hiebeler, D. E., 2007. Competing populations on fragmented landscapes with spatially structured heterogeneities: Improved landscape generation and mixed dispersal strategies. Journal of Mathematical Biology 54 (3), 337-356.

Hiebeler, D. E., Michaud, I. J., 2012. Quantifying spatial and temporal variability of spatially correlated disturbances. Ecological Modelling 240, 64-73.

Hiebeler, D. E., Michaud, I. J., Wasserman, B. A., Buchak, T. D., 2013. Habitat association in populations on landscapes with continuous-valued heterogeneous habitat quality. Journal of Theoretical Biology 317, 47-54.

Hiebeler, D. E., Morin, B. R., 2007. The effect of static and dynamic spatially structured disturbances on a locally dispersing population. Journal of Theoretical Biology 246 (1), 136-144.

Hodgson, J. A., Moilanen, A., Thomas, C. D., 2009. Metapopulation responses to patch connectivity and quality are masked by successional habitat dynamics. Ecology 90 (6), 1608-1619.

Iwasa, Y., 1991. Pessimistic plant: Optimal growth schedule in stochastic environments. Theoretical Population Biology 40, 246-268. 
Jacquemyn, H., Butaye, J., Hermy, M., 2003. Influence of environmental and spatial variables on regional distribution of forest plant species in a fragmented and changing landscape. Ecography 26 (6), 768-776.

Johst, K., Brandl, R., Eber, S., 2002. Metapopulation persistence in dynamic landscapes: The role of dispersal distance. Oikos 98, 263-270.

Johst, K., Drechsler, M., 2003. Are spatially correlated or uncorrelated disturbance regimes better for the survival of species? Oikos 103, 449-456.

Kallimanis, A., Kunin, W., Halley, J., Sgardelis, S., Apr. 2005. Metapopulation extinction risk under spatially autocorrelated disturbance. Conservation Biology 19 (2), 534-546.

Kerr, J. T., Pindar, A., Galpern, P., Packer, L., Potts, S. G., Roberts, S. M., Rasmont, P., Schweiger, O., Colla, S. R., Richardson, L. L., Wagner, D. L., Gall, L. F., Sikes, D. S., Pantoja, A., Jul. 2015. Climate change impacts on bumblebees converge across continents. Science 349 (6244), 177-180.

Keymer, J. E., Marquet, P. A., Vlasco-Hernández, J. X., Levin, S. A., Nov. 2000. Extinction thresholds and metapopulation persistence in dynamic landscapes. The American Naturalist 156 (5), 478-494.

Kie, J. G., Bowyer, R. T., Nicholson, M. C., Boroski, B. B., Loft, E. R., 2002. Landscape heterogeneity at differing scales: Effects on spatial distribution of mule deer. Ecology 83 (2), 530-544.

Kubo, T., Iwasa, Y., Furumoto, N., 1996. Forest spatial dynamics with gap expansion: Total gap area and gap size distribution. Journal of Theoretical Biology 180, 229-246.

Kun, Á., Oborny, B., Dieckmann, U., 2009. Intermediate landscape disturbance maximizes metapopulation density. Landscape Ecology 24, 1341-1350.

Lande, R., Engen, S., Sæther, B.-E., Sep. 1999. Spatial scale of population synchrony: Environmental correlation versus dispersal and density regulation. The American Naturalist 154 (3), 271-281. 
Langellotto, G. A., Denno, R. F., 2001. Benefits of dispersal in patchy environments: Mate location by males of a wing-dimorphic insect. Ecology 82 (7), 1870-1878.

Levin, S. A., Durrett, R., 1996. From individuals to epidemics. Philosophical Transactions: Biological Sciences 351, 1615-1621.

Liao, J., Li, Z., Hiebeler, D. E., Iwasa, Y., Bogaert, J., Nijs, I., 2013. Species persistence in landscapes with spatial variation in habitat quality: A pair approximation model. Journal of Theoretical Biology 335, 22-30.

Liao, J., Ying, Z., Hiebeler, D. E., Wang, Y., Takada, T., Nijs, I., 2015. Species extinction thresholds in the face of spatially correlated periodic disturbance. Scientific Reports 5, 15455.

Limberger, R., Wickham, S. A., 2012. Disturbance and diversity at two spatial scales. Oecologia 168, 785-795.

Lindborg, R., Eriksson, O., 2004. Historical landscape connectivity affects present plant species diversity. Ecology 85 (7), 1840-1845.

Linderman, M. A., An, L., Bearer, S., He, G., Ouyang, Z., Liu, J., 2006. Interactive effects of natural and human disturbances on vegetation dynamics across landscapes. Ecological Applications 16 (2), 452-463.

Loehle, C., Mar. 2007. Effect of ephemeral stepping stones on metapopulations on fragmented landscapes. Ecological Complexity 4 (1-2), 42-47.

Manrubia, S. C., Solé, R. V., 1997. On forest spatial dynamics with gap formation. Journal of Theoretical Biology 187, 159-164.

Masaki, T., 2004. Effect of the shape of forest fragments on tree population dynamics. Plant Ecology 172, 275-286.

Matlack, G. R., 2005. Slow plants in a fast forest: Local dispersal as a predictor of species frequencies in a dynamic landscape. Journal of Ecology 93, 50-59. 
Matlack, G. R., Leu, N. A., Dec. 2007. Persistence of dispersal-limited species in structured dynamic landscapes. Ecosystems 10 (8), 1287-1298.

Matlack, G. R., Monde, J., 2004. Consequences of low mobility in spatially and temporally heterogeneous ecosystems. Journal of Ecology 92, 1025-1035.

McCarthy, M. A., Lindenmayer, D. B., 2000. Spatially-correlated extinction in a metapopulation model of Leadbeater's Possum. Biodiversity and Conservation $9,47-63$.

McPeek, M. A., Holt, R. D., Dec. 1992. The evolution of dispersal in spatially and temporally varying environments. The American Naturalist 140 (6), 1010-1027.

Minor, E. S., Gardner, R. H., 2011. Landscape connectivity and seed dispersal characteristics inform the best management strategy for exotic plants. Ecological Applications 21 (3), 739-749.

Moloney, K. A., Levin, S. A., 1996. The effects of disturbance architecture on landscape-level population dynamics. Ecology 77 (2), 375-394.

Mortelliti, A., Sozio, G., Driscoll, D. A., Bani, L., Boitani, L., Lindenmayer, D. B., 2014. Population and individual-scale responses to patch size, isolation and quality in the hazel dormouse. Ecosphere 5 (9), 107.

Munguia, P., Osman, R. W., Hamilton, J., Whitlatch, R., Zajac, R., 2011. Changes in habitat heterogeneity alter marine sessile benthic communities. Ecological Applications 21 (3), 925-935.

Munroe, D. K., Southworth, J., Tucker, C. M., 2002. The dynamics of land-cover change in western Honduras: Exploring spatial and temporal complexity. Agricultural Economics 27, 355-369.

Naeem, S., Colwell, R. K., 1991. Ecological consequences of heterogeneity of consumable resources. In: Kolasa, J., Pickett, S. T. (Eds.), Ecological Heterogeneity. Springer-Verlag, pp. 224-255. 
Neuhauser, C., 1998. Habitat destruction and competitive coexistence in spatially explicit models with local interactions. Journal of Theoretical Biology 193, 445-463.

North, A., Ovaskainen, O., Jul. 2007. Interactions between dispersal, competition, and landscape heterogeneity. Oikos 116 (7), 1106-1119.

Oborny, B., 1994. Growth rules in clonal plants and environmental predictability — a simulation study. Journal of Ecology 82, 341-351.

Ovaskainen, O., Hanski, I., 2003. Extinction threshold in metapopulation models. Annales Zoologici Fennici 39, 81-97.

Pedigo, L. P., Rice, M. E., 2014. Entomology and Pest Management. Waveland Press, Inc.

Perry, G. L., Sep. 2002. Landscapes, space and equilibrium: Shifting viewpoints. Progress in Physical Geography 26 (3), 339-359.

Pike, N., Tully, T., Haccou, P., Ferrière, R., 2004. The effect of autocorrelation in environmental variability on the persistence of populations: An experimental test. Proc. R. Soc. Lond. B 271, 2143-2148.

Radinger, J., Wolter, C., 2015. Disentangling the effects of habitat suitability, dispersal, and fragmentation on the distribution of river fishes. Ecological Applications 25 (4), 914-927.

Ringsby, T. H., Sæther, B.-E., Tufto, J., Jensen, N., Solberg, E. J., 2002. Asynchronous spatiotemporal demography of a house sparrow metapopulation in a correlated environment. Ecology 83 (2), 561-569.

Ripa, J., Ives, A. R., 2003. Food web dynamics in correlated and autocorrelated environments. Theoretical Population Biology 64, 369-384.

Robinson, G. R., Holt, R. D., Gaines, M. S., Hamburg, S. P., Johnson, M. L., Fitch, H. S., Martinko, E. A., Jul. 1992. Diverse and contrasting effects of habitat fragmentation. Science 257, 524-526. 
Rodríguez, N., 2015. On an integro-differential model for pest control in a heterogeneous environment. Journal of Mathematical Biology 70 (5), 1177-1206.

Roff, D. A., Nov. 1994. Habitat persistence and the evolution of wing dimorphism in insects. The American Naturalist 144 (5), 772-798.

Roland, J., Taylor, P. D., Apr. 1997. Insect parasitoid species respond to forest structure at different spatial scales. Nature 386, 710-713.

Roy, M., Pascual, M., Levin, S. A., 2004. Competitive coexistence in a dynamic landscape. Theoretical Population Biology 66, 341-353.

Schooley, R. L., Branch, L. C., 2007. Spatial heterogeneity in habitat quality and cross-scale interactions in metapopulations. Ecosystems 10, 846-853.

Seabloom, E. W., Bjørnstad, O. N., Bolker, B. M., Reichman, O., 2005. Spatial signature of environmental heterogeneity, dispersal, and competition in successional grasslands. Ecological Monographs 75 (2), 199-214.

Shachak, M., Brand, S., 1991. Relations among spatiotemporal heterogeneity, population abundance, and variability in a desert. In: Kolasa, J., Pickett, S. T. (Eds.), Ecological Heterogeneity. Springer-Verlag, pp. 202-223.

Silbernagel, J., 2003. Spatial theory in early conservation design: Examples from Aldo Leopold's work. Landscape Ecology 18, 635-646.

Smith, A. T., Gilpin, M., 1997. Spatially correlated dynamics in a pika metapopulation. In: Hanski, I. A., Gilpin, M. E. (Eds.), Metapopulation Biology: Ecology, Genetics, and Evolution. Academic Press, pp. 407-428.

Terry, A. J., Gourley, S. A., Oct. 2010. Perverse consequences of infrequently culling a pest. Bulletin of Mathematical Biology 72 (7), 1666-1695.

Topping, C., Sunderland, K., 1994. A spatial population dynamics model for lepthyphantes tenuis (araneae: Linyphiidae) with some simulations of the spatial and temporal effects of farming operations and land-use. Agriculture, Ecosystems and Environment 48, 203-217. 
Torgersen, C. E., Price, D. M., Li, H. W., McIntosh, B. A., 1999. Multiscale thermal refugia and stream habitat associations of chinook salmon in northeastern Oregon. Ecological Applications 9 (1), 301-319.

Turner, M. G., Oct. 2010. Disturbance and landscape dynamics in a changing world. Ecology 91 (10), 2833-2849.

Van den Brink, P. J., Baveco, J. H., Verboom, J., Heimbach, F., 2007. An individual-based approach to model spatial population dynamics of invertebrates in aquatic ecosystems after pesticide contamination. Environmental Toxicology and Chemistry 26 (10), 2226-2236.

Verheyen, K., Vellend, M., Calster, H. V., Peterken, G., Hermy, M., 2004. Metapopulation dynamics in changing landscapes: A new spatially realistic model for forest plants. Ecology 85 (12), 3302-3312.

Visconti, P., Elkin, C., 2009. Using connectivity metrics in conservation planning - when does habitat quality matter? Diversity and Distributions 15, 602-612.

Vreysen, M., Robinson, A., Hendrichs, J. (Eds.), 2007. Area-Wide Control of Insect Pests: From Research to Field Implementation. Springer.

Wilcox, C., Cairns, B. J., Possingham, H. P., 2006. The role of habitat disturbance and recovery in metapopulation persistence. Ecology 87 (4), 855-863.

Ye, X., Skidmore, A. K., Wang, T., Sep. 2014. Joint effects of habitat heterogeneity and species' life-history traits on population dynamics in spatially structured landscapes. PLoS ONE 9 (9), e107742.

\section{Appendix A. Derivation of ODEs}

Appendix A.1. Landscape equations

A differential equation for the proportion of suitable sites can be written:

$$
\begin{aligned}
\frac{d p[s]}{d t} & =p[u] r_{u s}-p[s] r_{s u} \\
& =(1-p[s]) r_{u s}-p[s] r_{s u}
\end{aligned}
$$


This has equilibrium

$$
p^{*}[s]=\frac{r_{u s}}{r_{s u}+r_{u s}} .
$$

When describing doublet probabilities for the landscape, there are four probabilities: $p[s s], p[s u], p[u s]$, and $p[u u]$. The symmetry assumption $p[s u]=p[u s]$ and the fact that all four probabilities must sum to one reduces this to two independent probabilities. We could use $p[s s]$ and $p[s u]$ to characterize the system. Instead, we use the relation $p[s s]+p[s u]=p[s]$, and describe the system via $p[s]$ and $p[s s]$.

A system of ordinary differential equations describing the landscape dynamics consists of Equation (A.1), along with:

$$
\frac{d p[s s]}{d t}=2 p[s u] r_{u s}-2 p[s s] r_{s u}(1-\theta) .
$$

The system may be closed by making the substitution $p[s u]=p[s]-p[s s]$, which follows from Equation (2).

The equilibrium value of $p[s s]$ is

$$
p^{*}[s s]=\frac{2 r_{u s}^{2}}{2 r_{u s}^{2}+4 r_{u s} r_{s u}+2 r_{s u}^{2}-r_{s u}^{2} \theta-r_{s u} r_{u s} \theta} .
$$

When describing triplet probabilities for the landscape, there are eight probabilities. Symmetry allows two to be eliminated $(p[u s s]=p[s s u]$ and $p[u u s]=$ $p[s u u])$; the probabilities summing to one allows elimination of another one. Equation (2) implies $p[s u s]+p[s u u]=p[s s u]+p[u s u]$ (with both sums being equal to $p[s u]$ ), allowing one more probability to be eliminated, bringing the 


$$
\begin{aligned}
\frac{d p[u u u]}{d t}= & (2 p[u u s]+p[u s u]) r_{s u}+\frac{2 p[u s s](b-1) r_{s u}}{b} \\
& +\frac{1_{b>1}(p[s s s]+p[s u s])(b-2) r_{s u}}{b}-3 p[u u u] r_{u s} \\
\frac{d p[u u s]}{d t}= & \frac{r_{s u}}{b}\left\{1_{b>1}(p[s s s]+2 p[s u s])+p[u s s]\right\}+1_{b=1} p[s u s] r_{s u}+p[u u u] r_{u s} \\
& -p[u u s]\left(2 r_{u s}+r_{s u}\right) \\
\frac{d p[u s u]}{d t}= & \frac{p[u s s] 2 r_{s u}}{b}+p[u u u] r_{u s}-p[u s u]\left(2 r_{u s}+r_{s u}\right) \\
\frac{d p[u s s]}{d t}= & r_{u s}(p[u s u]+p[u u s])+\frac{p[s s s] r_{s u}}{b}-p[u s s]\left(r_{u s}+\frac{r_{s u}(b+1)}{b}\right)(\text { A.8) }
\end{aligned}
$$

The factor of $(b-1) / b$ in the second term of Equation (A.5) above comes from the fact that there are $(b-1) b$ locations that the upper-left corner of a $b \times b$ disturbance block could hit (each with rate $r_{s u} / b^{2}$ ) that would cause a [uss] triplet of sites to become a $[u u u]$ triplet. Similarly, the factor of $(b+1) / b$ in the last term of Equation (A.8) follows from the fact that there are $(b+1) b$ sites a disturbance block could hit which would cause the [uss] triplet to change its state to either $[u u u],[u u s]$, or $[u s u]$.

This could be further reduced to a system of two equations, if we assume that $p[s]$ and $p[s s]$ are already at their equilibrium values given by Equations (A.2) and (A.4), respectively. Note that the system of ODEs differs according to whether $b>1$ or $b=1$. Indicator functions are used to select terms according to the two cases, with $1_{b>1}$ being 1 or 0 according to whether $b$ is or is not larger than one. When $b=1$, at equilibrium, the landscape has no spatial correlations. However, during the transient dynamics before equilibrium, spatial correlations will exist if they were present initially; in that sense, the case where $b=1$ is therefore different from a model assuming everything is well-mixed.

Equations (1) and (2) may be used to make substitutions to make Equations (A.5)-(A.8) self-contained. When $b>1$, the system may be solved for the 
following equilibrium:

$$
\begin{aligned}
p^{*}[u u u]= & \frac{r_{s u}\left((1+b)(2+b) r_{s u}^{2}+\left(b-4+3 b^{2}\right) r_{s u} r_{u s}+2(b-2) b r_{u s}^{2}\right)}{\left(r_{s u}+r_{u s}\right)\left(r_{s u}+b r_{s u}+2 b r_{u s}\right)\left((2+b) r_{s u}+3 b r_{u s}\right)} \\
p^{*}[u u s]= & \frac{(1+b) r_{s u} r_{u s}\left((2+b) r_{s u}+b r_{u s}\right)}{\left(r_{s u}+r_{u s}\right)\left(r_{s u}+b r_{s u}+2 b r_{u s}\right)\left((2+b) r_{s u}+3 b r_{u s}\right)} \\
p^{*}[u s u]= & \frac{r_{s u} r_{u s}\left((1+b)(2+b)^{2} r_{s u}^{2}+b(2+b)(1+3 b) r_{s u} r_{u s}+2(b-2) b^{2} r_{u s}^{2}\right)}{\left(r_{s u}+r_{u s}\right)\left(r_{s u}+b r_{s u}+2 b r_{u s}\right)\left((2+b) r_{s u}+2 b r_{u s}\right)\left((2+b) r_{s u}+3 b r_{u s}\right)} \\
p^{*}[u s s]= & \frac{1}{\left(r_{s u}+r_{u s}\right)\left(r_{s u}+b r_{s u}+2 b r_{u s}\right)} \\
& \times \frac{2 b r_{s u} r_{u s}^{2}\left((2+b)^{2} r_{s u}+b(5+2 b) r_{u s}\right)}{\left((2+b) r_{s u}+2 b r_{u s}\right)\left((2+b) r_{s u}+3 b r_{u s}\right)} .
\end{aligned}
$$

When $b=1$, at equilibrium the system has no spatial correlations among adjacent sites, giving simply

$p^{*}[u u u]=(p[u])^{3}, \quad p^{*}[u u s]=p^{*}[u s u]=(p[u])^{2} p[s], \quad p^{*}[u s s]=p[u](p[s])^{2}$.

Note that in this model, the transition rates for the configurations of habitat types in any block of sites are fixed, and not dependent on the states of neighboring sites or on the prevalence of habitat types in the landscape. Because of that, the system of equations for the probabilities of habitat configurations for blocks (of any size) of sites will be a linear system of ODEs. The equilibrium distribution of habitat types for any size blocks is therefore analytically obtainable, in principle, as the solution to a system of linear equations.

\section{Appendix A.2. Doublet equations}

With three states per site, there are nine doublet probabilities $P[i j]$. However, one probability (say, $P[22]$ ) may be eliminated because all nine must sum to one by Equation (1). Rotational symmetry allows the elimination of three more probabilities via $P[i j]=P[j i]$ for $[i j] \in\{[01],[02],[12]\}$. The assumption that the landscape is at dynamic equilibrium means that both $P[11]$ and $P[1]=P[01]+P[11]+P[21]$ are fixed, allowing two more probabilities to be eliminated. That leaves a total of three independent probabilities. 
We choose to write ODEs for $P[00], P[01]$, and $P[02]$ :

$$
\begin{aligned}
\frac{d P[00]}{d t}= & 2 P[01] r_{u s}+2 P[02] \mu-P[00]\left(r_{s u}(2-\theta)+2 \frac{z-1}{z} \phi Q_{2 \mid 06} A .10\right) \\
\frac{d P[01]}{d t}= & (P[00]+P[02]) r_{s u}(1-\theta)+P[11] r_{u s}+P[21] \mu \\
& -P[01]\left(r_{u s}+r_{s u}+\frac{z-1}{z} \phi Q_{2 \mid 01}\right) \\
\frac{d P[02]}{d t}= & \frac{z-1}{z} P[00] \phi Q_{2 \mid 00}+P[12] r_{u s}+P[22] \mu \\
& -P[02]\left(\mu+r_{s u}(2-\theta)+\frac{\phi}{z}+\frac{z-1}{z} Q_{2 \mid 02}\right)
\end{aligned}
$$

We use ordinary pair approximation, assuming conditional independence between the states of the first and third sites in a triplet when conditioned on knowing the state of the second site, namely $Q_{i \mid j k}=P[i \cdot \cdot \mid \cdot j k] \approx P[i \cdot \cdot \mid \cdot j \cdot]=$ $Q_{i \mid j}=P[i j] / P[j]$. This is used to replace the quantities $Q_{2 \mid 00}, Q_{2 \mid 01}$, and $Q_{2 \mid 02}$ in the above ODEs.

The quantities $P[12]$ and $P[22]$ that appear in Equations (A.10)-(A.12) may be expressed in terms of known quantities by using Equations (4) and (5):

$$
\begin{aligned}
& P[12]=p^{*}[s]\left(1-q_{s \mid s}^{*}\right)-P[01] \\
& P[22]=p^{*}[s] q_{s \mid s}^{*}-P[00]-2 P[02] .
\end{aligned}
$$

Making all of the above substitutions in Equations (A.10)-(A.12) yields the system of Equations (11)-(13).

\section{Appendix A.3. Landscape-improved doublet equations}

Equations (A.10)-(A.12) include the quantities $Q_{2 \mid 00}, Q_{2 \mid 01}$, and $Q_{2 \mid 02}$, involving conditional probabilities that the first site in a triplet is suitable and occupied, given information about the states of the last two sites. Ordinary pair approximation conditionally neglects correlations between the first and third site, conditioned on knowing the state of the second. In the landscapeimproved pair approximation, we first compute the probability that the first site in the triplet is suitable given the known habitat types of the last two, and then compute the probability that the first site is occupied given that it's suitable 
(and given the state of the second site). In this way, information about habitat structure at the triplet scale is included (and, as mentioned in Section Appendix A.1, no approximations are needed when computing these triplet probabilities); population clustering is still utilized at the doublet scale.

Mathematically, this approach consists of the following approximations:

$$
\begin{aligned}
Q_{2 \mid 00} & \approx q_{s \mid s s} Q_{2 \cdot \mid s 0} \\
Q_{2 \mid 01} & \approx q_{s \mid s u} Q_{2 \cdot \mid s 0} \\
Q_{2 \mid 02} & \approx q_{s \mid s s} Q_{2 \cdot \mid s 0} .
\end{aligned}
$$

The common factor in all three expressions above can be written as

$$
Q_{2 \cdot \mid s 0}=\frac{P[20]}{P[s 0]}=\frac{P[20]}{P[00]+P[20]} .
$$

The other quantities in the above expressions can be expressed as

$$
\begin{aligned}
q_{s \mid s s} & =\frac{p[s s s]}{p[s s]}=\frac{p[s s]-p[u s s]}{p[s s]} \\
q_{s \mid s u} & =\frac{p[s s u]}{p[s u]}=\frac{p[u s s]}{p[s]-p[s s]}
\end{aligned}
$$

where $p[s], p[s s]$, and $p[u s s]$ may be substituted in from Equations (A.2), (A.4), and (A.9) at equilibrium.

Appendix A.4. Block equations

The rate of change of the probability for a general $2 \times 2$ block may be written as

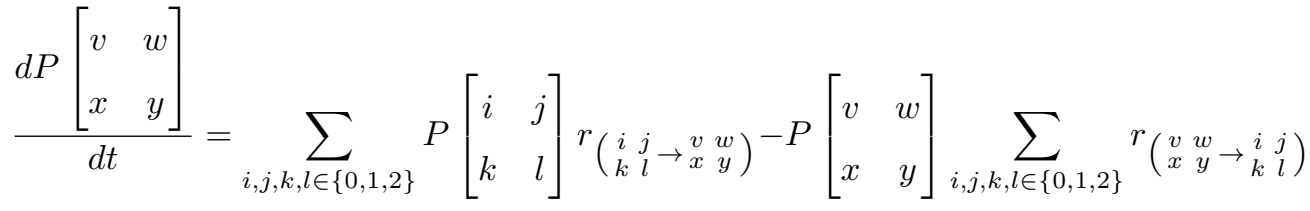

where $r_{\left(\begin{array}{ll}v & w \\ x & y\end{array} \rightarrow \begin{array}{ll}i & j \\ k & l\end{array}\right)}$ is the rate at which a $\left[\begin{array}{ll}v & w \\ x & y\end{array}\right]$ block transitions to a $\left[\begin{array}{ll}i & j \\ k & l\end{array}\right]$ block. We compute these transition rates according to how many sites are affected by the event causing the transition, as follows. 
A single-site transition may be a death, disturbance, or birth, with rates of those three events given below:

$$
\begin{aligned}
& r\left(\begin{array}{ll}
2 & w \\
x & y
\end{array} \rightarrow \begin{array}{ll}
0 & w \\
x & y
\end{array}\right)=\mu \\
& r\left(\begin{array}{lll}
v & w \\
x & y
\end{array} x_{x} \begin{array}{ll}
1 & w
\end{array}\right)=\frac{r_{s u}}{b^{2}} \quad \text { where } v \neq 1
\end{aligned}
$$

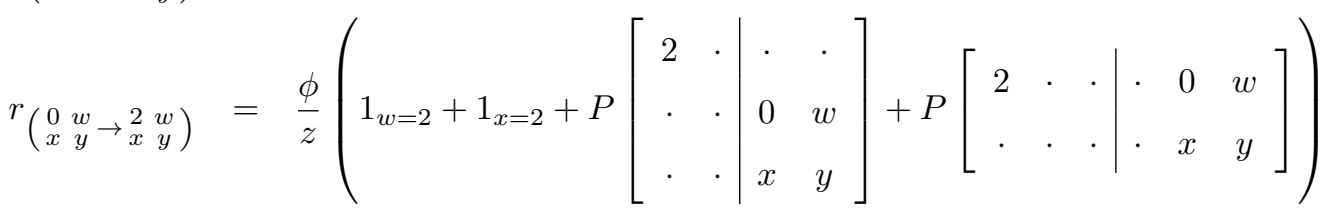

$$
\begin{aligned}
& \approx \frac{\phi}{z}\left(1_{w=2}+1_{x=2}+P\left[\begin{array}{cc|cc}
2 & \cdot & \cdot & \cdot \\
\cdot & \cdot & 0 & w
\end{array}\right]+P\left[\begin{array}{cc|cc}
2 & \cdot & \cdot & 0 \\
\cdot & \cdot & \cdot & x
\end{array}\right]\right) \\
& =\frac{\phi}{z}\left(1_{w=2}+1_{x=2}+\frac{P\left[\begin{array}{cc}
2 & \cdot \\
0 & w
\end{array}\right]}{P[0 w]}+\frac{P\left[\begin{array}{cc}
2 & 0 \\
\cdot & x
\end{array}\right]}{P[0 x]}\right) \text {. }
\end{aligned}
$$

During a birth event, the top-left corner may be colonized by one of its known neighbors in the block, if either of them are in state 2 (occupied), or by one of its two neighbors outside of the block. When approximating the probability that a particular one of those external neighbors is occupied, we again neglect correlations between non-adjacent sites in the lattice, conditioned on knowing the state of a site between them. The probabilities of the partial blocks above may be obtained by summation, as in Equation (3).

Note that in the disturbance transition given by Equation (A.13) above, we ignore disturbance events that hit multiple sites but only actually change the state of one site (for example, if a disturbance affects all four sites in the block, but $w=x=y=1$, then those three sites do not change state). Events hitting multiple sites are counted below.

A two-site transition may only be a disturbance, since intrinsic mortality and birth only affect one site at a time. Two-site disturbances can only affect two adjacent sites in the block; they cannot affect two diagonally opposite sites 
889

within the block. The rates of these events are:

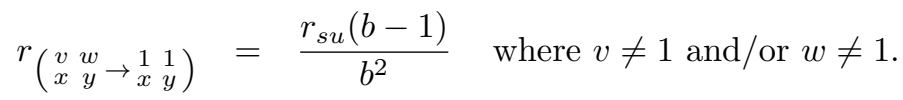

890

The above rate counts the fact that there are $b-1$ locations a disturbance block could be focused at which would affect only the top two sites in the block.

No events can affect exactly three sites within a $2 \times 2$ block, because disturbance events are square. Finally, a four-site transition may only be a disturbance, with the following rate:

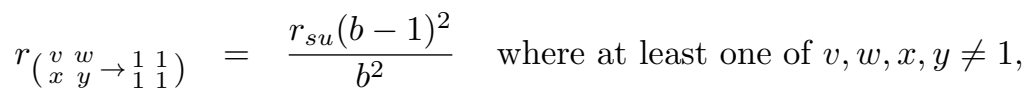

895 again counting the number of locations a disturbance block could target which would affect all four sites in the $2 \times 2$ block being considered. 